\title{
Racial disparity in kidney transplant survival relates to late rejection and is independent of steroid withdrawal
}

\author{
Angela Liu $^{1}$ | Kenneth J. Woodside ${ }^{2}$ (D) | Joshua J. Augustine ${ }^{3}$ | Nagaraju Sarabu ${ }^{1,4}$
}

\author{
${ }^{1}$ Case Western Reserve University School of \\ Medicine, Cleveland, Ohio \\ ${ }^{2}$ Department of Surgery, University of \\ Michigan, Ann Arbor, Michigan \\ ${ }^{3}$ Department of Nephrology, Cleveland \\ Clinic, Cleveland, Ohio \\ ${ }^{4}$ Division of Nephrology, Department of \\ Medicine, University Hospitals Cleveland \\ Medical Center, Cleveland, Ohio
}

\section{Correspondence}

Nagaraju Sarabu, University Hospitals Cleveland Medical Center, Cleveland, $\mathrm{OH}$. Email: nagaraju.sarabu@uhhospitals.org

\begin{abstract}
Black kidney transplant recipients have more acute rejection (AR) and inferior graft survival. We sought to determine whether early steroid withdrawal (ESW) had an impact on AR and death-censored graft loss (DCGL) in blacks. From 2006 to 2012, AR and graft survival were analyzed in 483 kidney recipients ( 208 black and 275 nonblack). Rates of ESW were similar between blacks (65\%) and non-blacks (67\%). AR was defined as early ( $\leq 3$ months) or late ( $>3$ months). The impact of black race, early $A R$, and late $A R$ on death-censored graft failure was analyzed using univariate and multivariate Cox models. Blacks had greater dialysis vintage, more deceased donor transplants, and less HLA matching, yet rates of early AR were comparable between blacks and non-blacks. However, black race was a risk factor for late AR (HR: 3.48 (95\% Cl: 1.87-6.47)) Blacks had a greater rate of DCGL, partially driven by late AR (HR with late AR: 5.6; 95\% Cl: 3.3-9.3). ESW had no significant interaction with black race for risk of early AR, late AR, or DCGL. Independent of ESW, black kidney recipients had a higher rate of late AR after kidney transplantation. Late AR was highly predictive of DCGL and contributed to inferior graft survival in blacks.
\end{abstract}

\section{KEYWORDS}

acute rejection, African American, early steroid withdrawal, graft survival, kidney transplant

\section{1 | INTRODUCTION}

Black kidney transplant recipients have had inferior allograft survival that continues in the modern era of kidney transplantation. ${ }^{1-3}$ This difference is, in part, related to access to transplantation, with discrepancies in living donor rates and dialysis vintage, ${ }^{4}$ delays in listing for transplantation, ${ }^{5}$ greater rates of deceased and marginal donor kidneys, ${ }^{6,7}$ and less favorable HLA matching. ${ }^{8}$ Such discrepancies contribute to a greater rate of $A R$ in black recipients which impacts upon allograft survival. ${ }^{2}$

The prognosis for kidney survival after AR appears to vary depending on the timing of the rejection episode. Late AR is typically defined in the literature as occurring after the first 90 day post-transplantation and has been shown to have a greater negative impact on graft survival. ${ }^{9-12}$ One registry analysis showed similar outcomes in blacks in the early post-transplant period, but greater rates of late AR and late allograft failure in blacks. ${ }^{13}$ Immunosuppressive status, including early steroid withdrawal (ESW), was not analyzed in that study.

Because of greater rates of AR in blacks, ESW in this subgroup has remained controversial. The Astellas Corticosteroid Withdrawal Study showed a similar rate of biopsy proven rejection in blacks with and without $\mathrm{ESW},{ }^{14}$ but there were trends in greater chronic changes in blacks after ESW. A recent analysis of data from the United Network of Organ Sharing (UNOS) showed no increase in AR or allograft loss in a subset of black recipients who underwent ESW, ${ }^{15}$ providing that patients were carefully selected and received depleting antibody induction therapy with tacrolimus and mycophenolate mofetil therapy. In the study herein, we observed that black recipients had comparable rates of early AR but greater rates of late AR, consistent with registry data. We sought to further determine whether ESW influenced the higher rate of late AR seen in black recipients, and to analyze the impact of early and late AR, black race, and ESW on allograft survival. 
TAB LE 1 Baseline characteristics of kidney transplant recipients $(n=483)$

\begin{tabular}{|c|c|c|c|}
\hline & Blacks (208) & Non-blacks (275) & $P$-value \\
\hline $\begin{array}{l}\text { Age at } \\
\text { transplant }\end{array}$ & $48.3(12)$ & $50.5(13.6)$ & 0.1 \\
\hline Female & $78(37.5)$ & $109(39.6)$ & 0.7 \\
\hline $\begin{array}{l}\text { Dialysis vintage } \\
\text { (mo) }\end{array}$ & $65.8(39.1)$ & $28.2(33.2)$ & $<0.001$ \\
\hline $\begin{array}{l}\text { Baseline } \\
\text { diabetes }\end{array}$ & $70(33.7)$ & $82(29.8)$ & 0.4 \\
\hline $\begin{array}{l}\text { Prior } \\
\text { transplant }\end{array}$ & $18(8.7)$ & 38 (13.8) & 0.1 \\
\hline $\begin{array}{l}\text { Deceased } \\
\text { donor }\end{array}$ & $177(85.1)$ & $131(47.6)$ & $<0.001$ \\
\hline Donor age & $43.3(15.4)$ & $43.9(13.3)$ & 0.7 \\
\hline HLA mismatch & $4.51(1.46)$ & $3.60(1.92)$ & $<0.001$ \\
\hline Class I PRA & 17. (30.7) & $12.5(25.2)$ & 0.1 \\
\hline Class II PRA & $9.0(23.1)$ & $10.1(25.2)$ & 0.6 \\
\hline ATG induction & $179(86.1)$ & $152(55.3)$ & $<0.001$ \\
\hline $\begin{array}{l}\text { Delayed graft } \\
\text { function }\end{array}$ & 34 (16.3) & $50(18.2)$ & 0.7 \\
\hline $\begin{array}{r}\text { Early steroid } \\
\text { withdrawal }\end{array}$ & $135(64.9)$ & $185(67.3)$ & 0.7 \\
\hline
\end{tabular}

\section{2 | METHODS}

\section{1 | Study cohort and immunosuppression}

A retrospective analysis of transplant recipients at University Hospitals Cleveland Medical Center was conducted following Institutional Review Board approval. Between January, 2006 and December, 2012, 500 consecutive patients received solitary kidney transplants. Patients were identified as black or non-black based on description in the demographic section of the medical records. For the purposes of this study, non-black included all other races and ethnicities. All patients received induction therapy with either $20 \mathrm{mg}$ of basiliximab on postoperative days 0 and 4 or antithymocyte globulin (ATG) at a cumulative dosage of $6 \mathrm{mg} / \mathrm{kg}$. Per our center protocol, all deceased donor (DD) kidney recipients received ATG, whereas living donor recipients, who were older than 50 years, received basiliximab. All patients were treated with oral tacrolimus therapy, typically starting within 24 hours of transplantation, with a target level of 8-12 ng/mL in the first 3 months, followed by a target of $5-8 \mathrm{ng} / \mathrm{mL}$ after 3 months. All patients also received antiproliferative therapy with mycophenolic acid derivatives. Intravenous methylprednisolone was administered at a $250 \mathrm{mg}$ dose in the operating room, followed by $60 \mathrm{mg}$ twice daily for 4 days. After postoperative day 4 , ESW was undertaken in $65 \%$ of patients overall. Primary reasons for not withdrawing steroids in the remaining cohort included delayed graft function (DGF) in $43 \%$, high antibody sensitization with a panel reactive antibody (PRA) class I or II of $\geq 80 \%$ at the time of transplant in $20 \%$, and prior kidney transplantation in $28 \%$.

\section{2 | Acute rejection}

Acute rejection episodes were identified by biopsy for cause and treated with pulse dose corticosteroids or ATG therapy for cellular rejection, or in cases of antibody-mediated rejection (AMR), with corticosteroids, plasmapheresis, intravenous immunoglobulin, and/or bortezomib. As in previous studies, $9,12,16$ early AR was defined as occurring within 3 months of transplantation and late AR after 3 months. Data were recorded on the presence of acute/ active AMR on biopsy based on BANFF criteria contemporary to the biopsy, along with the presence of donor-specific antibodies present in circulation and with either positive peritubular capillary C4d staining diffusely by immunofluorescence or the presence of peritubular capillaritis. ${ }^{17}$ Nonadherence was evaluated and recorded if patients were described as nonadherent by the clinical transplant nephrologists or nurse coordinators in the patient medical record.

\section{3 | Statistical analyses}

Values are shown as mean $\pm \mathrm{SD}$, median, or percentage. Comparisons for baseline variables were made between black and non-black recipients. Comparisons were performed using a $t$ test for continuous variables and a Pearson chi-square test for dichotomous variables. Additional variables examined included recipient factors (age, gender, diabetes, dialysis vintage, prior transplant, PRA), transplant, and donor factors (donor source, donor age, HLA mismatch, DGF, induction immunosuppression, and ESW). We assessed association of black race with early $A R$, and late $A R$ using Cox regression models. To assess interaction between black race and ESW, we fit Cox regression models with an interaction term with black race. In addition, since blacks and non-blacks were different in other baseline variables, we included variables whose $P$-values were $<0.2$ in multivariable models.

To compare time from date of transplant to acute rejection (either early or late) between blacks and non-blacks, we fit Kaplan-Meier curves to rejection-free survival and used log-rank test to compare both distributions. Patients were censored at time of death, last follow-up date, or November 30, 2015, which was the end of the study period. For time to death-censored graft loss (DCGL), patients were again censored at time of death, last follow-up date, or November 30,2015 . Kaplan-Meier survival curves were generated with time to DCGL, stratified by black race vs. non-black race. Log-rank test was used to compare the survival distributions of DCGL between blacks and non-blacks. Since early AR and late AR occurred during the follow-up period, these variables were considered as time-varying covariates in survival analyses. Extended Kaplan-Meier curves using Snappin method were generated to examine time to DCGL, stratified by early $A R$ vs. no early $A R$, and late $A R$ vs. no late $A R$. To examine the effect of black race on DCGL, we fit Cox regression models. To examine the effect of early AR and late AR on DCGL, we fit extended Cox regression models with these predictors as time-varying covariates. All analyses were performed using R.3.5.1 ${ }^{\circledR}$. 


\section{3 | RESULTS}

A total cohort of 500 patients received solitary kidney transplants during the study period. Seventeen patients with primary nonfunction or early death (<30 days) were excluded from analysis. Baseline characteristics are shown in Table 1. Blacks made up $43 \%$ of the cohort overall. Compared to non-blacks, blacks had longer dialysis vintage, a higher rate of deceased donor transplant, greater HLA mismatching, and a higher rate of ATG induction therapy. Rates of ESW were similar between non-blacks (67\%) and blacks (65\%).

\subsection{Early acute rejection}

Forty-eight patients (9.9\%) among the remaining cohort of 487 patients experienced early AR in the first 3 months of kidney transplant. Of these, 19 (9.1\%) occurred in blacks and 29 (10.5\%) occurred in non-blacks (OR for black vs. non-black: 0.86 (95\% Cl: 0.48-1.54)). ESW was associated with a $38 \%$ higher risk of early $A R$, but this was not statistically significant (HR: 1.38 (95\% Cl: 0.73-2.61)). Also, there was no significant interaction between black race and ESW. In a multivariable model, after adjusting for other variables, black race was not significantly associated with early AR (Table 2 ).

\section{2 | Late acute rejection}

Forty-nine patients (10.1\%) among the whole cohort experienced late AR after the first 3 months of kidney transplant. Of these, 35 (16.8\%) occurred in blacks and 14 (5.1\%) occurred in non-blacks. Black race was associated with a fourfold increase in risk of late AR, and this was statistically significant (HR: 3.48 (95\% Cl: 1.87-6.47)). ESW was associated with a $28 \%$ less likelihood of late AR but this was not statistically significant (HR: 0.72 (95\% Cl: 0.40-1.28)). As with early $A R$, there was no significant interaction between black race and ESW for risk of late AR. In a multivariable model, after adjusting for other variables, black race was significantly associated with late AR (Table 2).

Figure 1 illustrates Kaplan-Meier curves for freedom from rejection (both early $A R$ and late $A R$ ) survival, stratified by black race. Blacks had higher rates of late AR at all time points, with a notable increase after 2 year post-transplantation. We also explored rates of AMR. Not surprisingly, AMR on biopsy was more common in late $A R$, occurring in $33 \%$ of cases compared to $8 \%$ of early $A R$

TAB LE 2 Hazard ratios for early and late AR by black race

\begin{tabular}{|lll}
\hline Model & Early AR & Late AR \\
\hline Black race, unadjusted & $0.86(0.48-1.54)$ & $3.48(1.87-6.47)$ \\
\hline $\begin{array}{l}\text { Black race, adjusted for } \\
\text { Steroid withdrawal }\end{array}$ & $0.87(0.49-1.55)$ & $3.44(1.96-7.19)$ \\
\hline $\begin{array}{c}\text { Black race, adjusted for } \\
\text { others }^{\text {a }}\end{array}$ & $0.89(0.44-1.78)$ & $2.28(1.10-5.41)$ \\
\hline
\end{tabular}

${ }^{a}$ Adjusted for steroid withdrawal, age at transplant, dialysis vintage, donor source, class I flow PRA, ATG induction, HLA mismatch.
$(P=0.003) . A M R$ occurred in $29 \%$ of blacks with late AR vs. $43 \%$ of non-blacks with $\operatorname{AMR}(P=0.13)$. In patients with early $A R$, five patients $(10 \%)$ were described as nonadherent, including two patients who displayed nonadherence after the early AR episode. Alternatively, twelve patients (24\%) were deemed nonadherent in the late AR cohort ( $P=0.07$ vs early AR). Black patients with late AR had documented nonadherence in $29 \%$ of cases vs. $14 \%$ of cases in non-blacks $(P=0.30)$.

\section{3 | Graft survival}

A total of 117 out of 483 (24\%) patients in the cohort had overall graft failure (including death with function) during a mean follow-up of 5 years 3 months. Overall graft survival was $97 \%$ at 1 year, $80 \%$ at 5 years, and $65 \%$ at 10 years. Since we were interested in interaction between ESW and black race, and ESW would mainly affect death-censored graft survival (DCGS), we focused on DCGS for further analysis.

A total of 72 out of 483 (15\%) patients in the cohort had DCGL during a mean follow-up of 5 years 3 months. DCGS was $99 \%$ at 1 year, $87 \%$ at 5 years, and $78 \%$ at 10 years. Figure 2 illustrates Kaplan-Meier survival curves for DCGS, stratified by black race vs. non-blacks. DCGS was $99 \%$ at 1 year, $84 \%$ at 5 years, and $67 \%$ at 10 years for blacks compared with $98 \%$ at 1 year, $88 \%$ at 5 years, and $86 \%$ at 10 years for non-blacks $(P$-value $=0.01$ ). Black race was

\section{Rejection Free Survival, by Black Race}

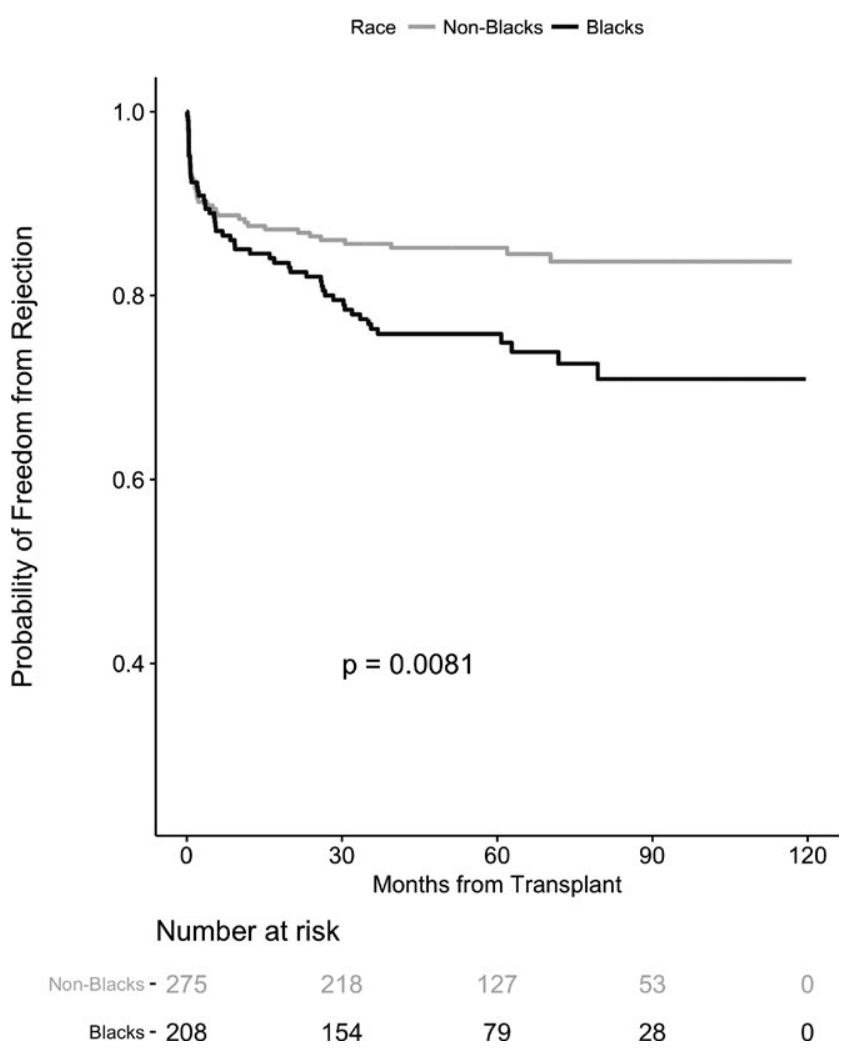

FIGURE 1 Kaplan Meier curves for rejection-free survival, stratified by black race 
Death Censored Graft Survival, by Black Race

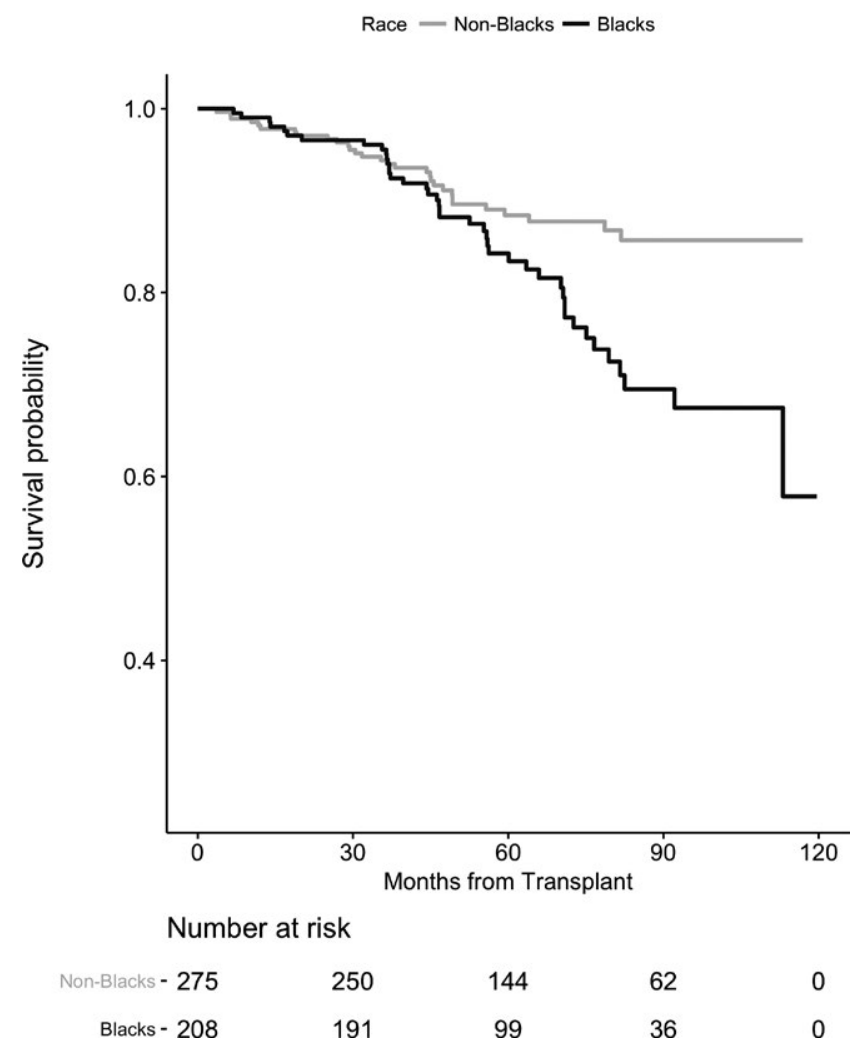

FIG URE 2 Kaplan Meier survival curves for death-censored graft survival (DCGS), stratified by black race

associated with a 91\% higher likelihood of DCGL (HR: 1.91; 95\% $\mathrm{Cl}$ : 1.19-3.05). Black race remained a risk factor for DCGL after adjustment for ESW (HR: 1.84; 95\% Cl: 1.15-2.95). Figure 3 illustrates Kaplan-Meier survival curves for DCGS, stratified by early AR. Early AR was associated with a 2.3-fold increased risk of DCGL (HR: 2.3; 95\% Cl: 1.4-3.9). Figure 4 illustrates Kaplan-Meier survival curves for DCGS, stratified by late AR. Late AR was much stronger risk factor for DCGL than early AR (HR: 5.6; 95\% Cl: 3.3-9.3). In the multivariable Cox model after adjustment for early AR, hazard of black race for DCGL was 1.9 (95\% Cl: 1.2-2.9). In the multivariate Cox model after adjustment for late AR, hazard ratio of black race for DCGL was 1.8 (95\% Cl: 1.1-2.7). However, after adjustment for other variables, black race was no longer a significant risk factor for DCGL (Table 3).

\section{4 | DISCUSSION}

In this analysis of long-term outcomes in a large cohort of kidney transplant recipients with ESW, we examined outcomes related to early and late AR and allograft survival in black vs. non-black recipients. Blacks were disadvantaged, with higher rates of deceased donor transplants, longer dialysis vintage, and greater HLA mismatching. Despite these differences, early AR rates were similar between blacks and non-blacks. There was a higher rate of ATG induction in black recipients, which may have contributed to comparable early

\section{Death Censored Graft Survival, by Early AR}

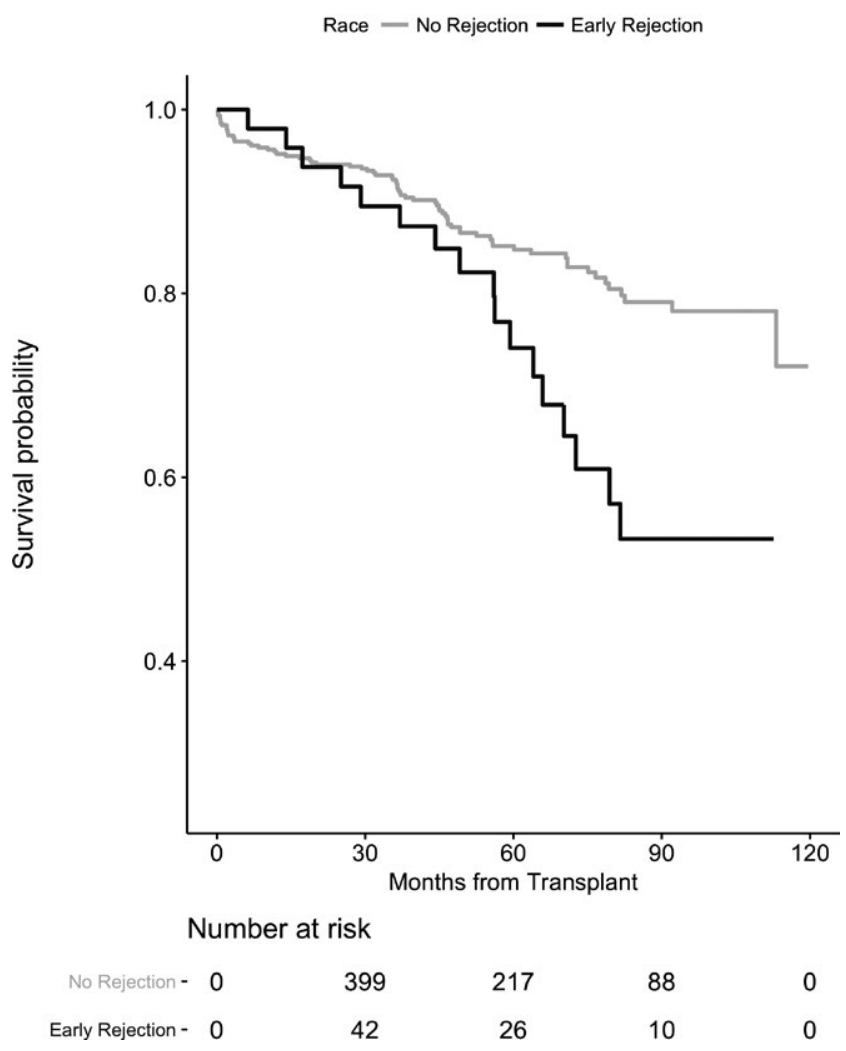

FIGURE 3 Kaplan Meier survival curves for death-censored graft survival (DCGS), stratified by early AR

AR rates in black recipients. ATG may be protective particularly in patients undergoing ESW. The Astellas Corticosteroid Withdrawal Study showed a trend for less rejection after ESW in patients who received ATG compared to basiliximab, ${ }^{14}$ and a recent UNOS registry analysis showed a benefit in allograft survival with ATG specifically in ESW patients. ${ }^{18}$

We found an overrepresentation of black recipients with late AR compared with non-blacks. Rates appeared higher at all time points, with a spike in AR after 2 year post-transplantation in blacks. Late AR conveyed an approximately 5 -fold increase in the rate of DCGL. It is likely that late AR is a major factor contributing to the well-described discrepancies in allograft survival between blacks and non-blacks. ${ }^{19-21}$ A previous analysis of UNOS data compared outcomes between black and non-black recipients of paired DD kidneys. ${ }^{13}$ Black recipients in that analysis were actually shown to have superior overall early survival at 6 months in a multivariable model. However, there was a divergence in allograft survival beyond 1 year, with greater graft loss in blacks over time, and this late graft loss appeared to be driven by late AR.

ESW has been commonly employed in the modern transplant era, with the goal of reducing the long-term morbidity associated with steroid regimens. As this study is retrospective, patients were not randomized to ESW; higher risk patients with DGF, prior transplant, or high antibody sensitization were maintained on steroid therapy in our cohort. However, rates of ESW were similar in 


\section{Death Censored Graft Survival, by Late AR}

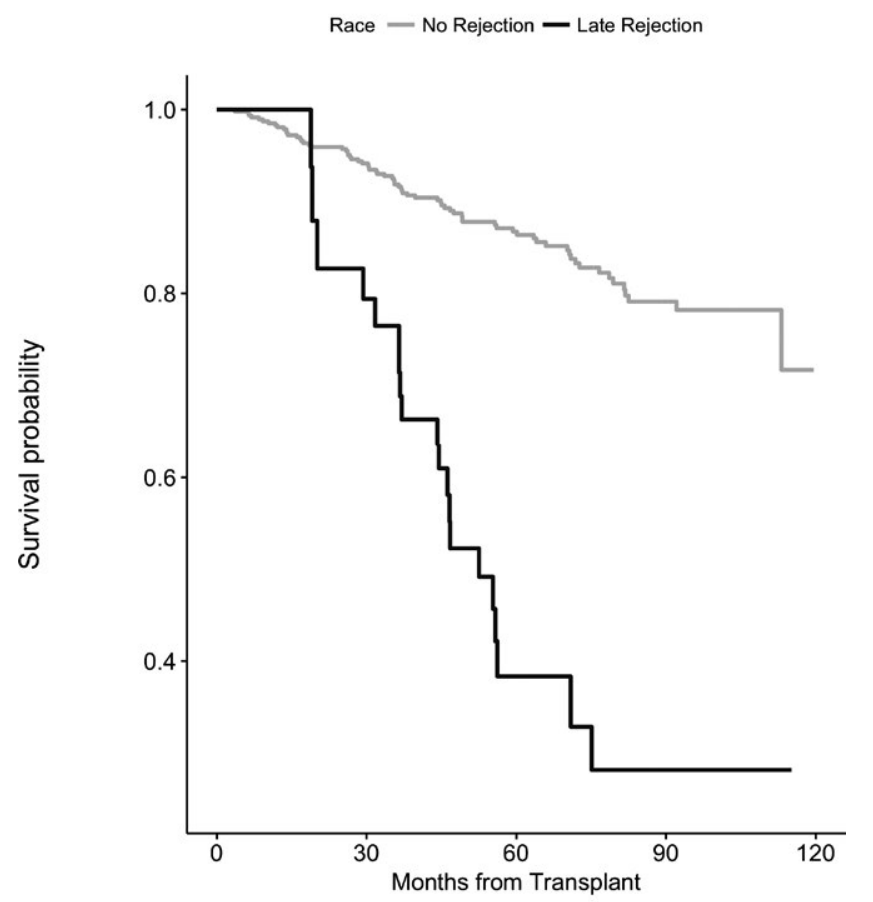

Number at risk

\begin{tabular}{|c|c|c|c|}
\hline No Rejection - 0 & 417 & 235 & 94 \\
\hline Late Rejection - 0 & 24 & 8 & 4 \\
\hline
\end{tabular}

FIGURE 4 Kaplan Meier survival curves for death-censored graft survival (DCGS), stratified by late $A R$

TAB LE 3 Cox model hazard ratios for death-censored graft failure

\begin{tabular}{|ll|}
\hline Model & $\begin{array}{l}\text { Death-censored } \\
\text { graft failure }\end{array}$ \\
\hline Black race, unadjusted & $1.91(1.19-3.05)$ \\
\hline Black race, adjusted for steroid withdrawal & $1.84(1.15-2.95)$ \\
\hline Black race, adjusted for others ${ }^{a}$ & $1.36(0.78-2.38)$ \\
\hline Black race, adjusted for early AR & $1.89(1.24-2.89)$ \\
\hline Black race, adjusted for late AR & $1.77(1.14-2.74)$ \\
\hline
\end{tabular}

${ }^{a}$ Adjusted for steroid withdrawal, age at transplant, dialysis vintage, donor source, class I flow PRA, ATG induction, HLA mismatch.

blacks and non-blacks. We found that ESW was associated with a trend for greater risk of early AR, but not late AR. Gaber and colleagues reported on a follow-up of the Astellas Corticosteroid Withdrawal Study and, after including cases of borderline rejection, identified a greater rate of early AR in ESW patients, similar to our findings. ${ }^{22}$ Interestingly, they found that late AR (beyond 2 years) in blacks was higher in steroid maintenance patients. We also found that ESW did not contribute to late AR in blacks. On the contrary, the highest rate of late AR was seen in blacks who maintained corticosteroid therapy. Black patients with steroid maintenance may have been identified early on as higher risk based on demographic or socioeconomic factors, which may explain this finding. Regardless, based on our ESW protocol, ESW did not appear to contribute to inferior outcomes seen in black recipients.

Other studies have identified late AR as a factor contributing to worse outcomes and lower rates of graft survival compared with early AR. Sijpkens et al found a marked decreased in longterm allograft survival associated with late $A R$, similar to our findings. ${ }^{12}$ In our study, we identified AMR in one-third of patients with late $A R$, with a greater than fourfold rate compared with early AR. This likely contributed to the high rate of allograft loss, as late AMR responds poorly to current therapy. ${ }^{9,23}$ In other studies, late AMR was previously found to be associated with younger age, suboptimal immunosuppression, and nonadherence to medical therapy. ${ }^{9}$

Nonadherence contributed to late AR in approximately one quarter of cases, with a trend for higher rates of nonadherence in blacks in our analysis. At later time points after transplantation, patients typically have less frequent follow-up and laboratory testing and may have variable rates of follow-up. Patients may also develop financial challenges in paying for medications over time. ${ }^{24}$ It is likely that socioeconomic factors play a role in the differences in late outcomes in black kidney recipients, as less education and lower rates of private insurance have been found to persist in blacks in the modern transplant era. ${ }^{1}$ As a result of the findings herein, we advocate for close patient monitoring, even years after transplantation, particularly in patients at higher risk of late AR. We did not analyze trough levels of tacrolimus in this cohort, but there may be a need for maintenance of higher targets above $5-8 \mathrm{ng} / \mathrm{mL}$ in higher risk patients as well. Variability in levels of tacrolimus has been shown to contribute to late AR and graft loss. ${ }^{25,26}$

This study has limitations. The study is retrospective, resulting in a selection bias for which patients are in the ESW group; patients were selected for ESW based on perceived risk and definite criteria, although black race was not considered an indication for avoidance of ESW. Analysis of AMR was also limited and descriptive in nature. Furthermore, nonadherence is very difficult to measure ${ }^{27,28}$ and relied on subjective interpretation of healthcare providers, which may be influenced by unconscious bias.

In conclusion, we demonstrate that inferior outcomes in blacks are driven by late outcomes, including late AR, and show that such late outcomes do not appear to be related to ESW. Solving the dilemma of heightened late AR in blacks may not rely on corticosteroid dosing, but rather on a multipronged approach of improved immunological monitoring, graft follow-up, and assessment of adherence late after transplantation, tailored to the individual patient's biological and socioeconomic characteristics. Prospective studies are needed to determine to what degree disparate late outcomes for black patients are driven by biologic or socioeconomic factors.

\section{CONFLICT OF INTEREST}

The authors declare no conflict of interests. 


\section{AUTHORS' CONTRIBUTIONS}

All authors: Participated in data analysis and article writing, editing, and revision; Angela Liu and Joshua J. Augustine: Contributed to research design and data collection.

\section{ORCID}

Kenneth J. Woodside (iD http://orcid.org/0000-0002-7495-3758

Nagaraju Sarabu (iD http://orcid.org/0000-0001-6084-8677

\section{REFERENCES}

1. Taber DJ, Egede LE, Baliga PK. Outcome disparities between African Americans and Caucasians in contemporary kidney transplant recipients. Am J Surg. 2017;213:666-672.

2. Gralla J, Le CN, Cooper JE, Wiseman AC. The risk of acute rejection and the influence of induction agents in lower-risk African American kidney transplant recipients receiving modern immunosuppression. Clin Transplant. 2014;28:292-298.

3. Gordon EJ, Ladner DP, Caicedo JC, Franklin J. Disparities in kidney transplant outcomes: a review. Semin Nephrol. 2010;30:81-89.

4. Joshi S, Gaynor JJ, Ciancio G. Review of ethnic disparities in access to renal transplantation. Clin Transplant 2012;26:E337-E343.

5. Johansen KL, Zhang R, Huang Y, Patzer RE, Kutner NG. Association of race and insurance type with delayed assessment for kidney transplantation among patients initiating dialysis in the United States. Clin J Am Soc Nephrol. 2012;7:1490-1497.

6. Tankersley MR, Gaston RS, Curtis JJ, et al. The living donor process in kidney transplantation: influence of race and comorbidity. Transplant Proc. 1997;29:3722-3723.

7. Chavalitdhamrong D, Gill J, Takemoto S, et al. Patient and graft outcomes from deceased kidney donors age 70 years and older: an analysis of the Organ Procurement Transplant Network/ United Network of Organ Sharing database. Transplantation. 2008;85:1573-1579.

8. Rebellato LM, Arnold AN, Bozik KM, Haisch CE. HLA matching and the United Network for Organ Sharing Allocation System: impact of HLA matching on African-American recipients of cadaveric kidney transplants. Transplantation. 2002;74:1634-1636.

9. Dörje $\mathrm{C}$, Midtvedt $\mathrm{K}$, Holdaas $\mathrm{H}$, et al. Early versus late acute antibody-mediated rejection in renal transplant recipients. Transplantation. 2013;96:79-84.

10. Koo EH, Jang HR, Lee JE, et al. The impact of early and late acute rejection on graft survival in renal transplantation. Kidney Res Clin Pract. 2015;34:160-164.

11. Matas AJ, Gillingham KJ, Payne WD, Najarian JS. The impact of an acute rejection episode on long-term renal allograft survival (t1/2). Transplantation. 1994;57:857-859.

12. Sijpkens YWJ, Doxiadis IIN, Mallat MJK, et al. Early versus late acute rejection episodes in renal transplantation. Transplantation. 2003;75:204-208.

13. Keith D, Patrie JT. Short-term kidney transplant outcomes among African American recipients do not predict long-term outcomes: donor pair analysis. Clin Transplant. 2011;25:69-76.

14. Woodle ES, First MR, Pirsch J, et al. . A prospective, randomized, double-blind, placebo-controlled multicenter trial comparing early
(7 day) corticosteroid cessation versus long-term, low-dose corticosteroid therapy. Ann Surg. 2008;248:564-577.

15. Taber DJ, Hunt KJ, Gebregziabher M, et al. A comparative effectiveness analysis of early steroid withdrawal in black kidney transplant recipients. Clin J Am Soc Nephrol. 2017;12:131-139.

16. Joseph JT, Kingsmore DB, Junor BJR, et al. The impact of late acute rejection after cadaveric kidney transplantation. Clin Transplant. 2001;15:221-227.

17. Haas M, Sis B, Racusen LC, et al. Banff 2013 meeting report: inclusion of c4d-negative antibody-mediated rejection and antibodyassociated arterial lesions. Am J Transplant. 2014;14:272-283.

18. Tanriover B, Jaikaransingh V, MacConmara MP, et al. Acute rejection rates and graft outcomes according to induction regimen among recipients of kidneys from deceased donors treated with tacrolimus and mycophenolate. Clin J Am Soc Nephrol. 2016;11:1650-1661.

19. Butkus DE, Meydrech EF, Raju SS. Racial differences in the survival of cadaveric renal allografts. Overriding effects of HLA matching and socioeconomic factors. N Engl J Med. 1992;327:840-845.

20. Eckhoff DE, Young CJ, Gaston RS, et al. Racial disparities in renal allograft survival: a public health issue? J Am Coll Surg. 2007;204:894-902.

21. Narayanan M, Pankewycz O, Shihab F, Wiland A, McCague K, Chan L. Long-term outcomes in African American kidney transplant recipients under contemporary immunosuppression: a four-yr analysis of the Mycophenolic acid Observational REnal transplant (MORE) study. Clin Transplant. 2014;28:184-191.

22. Gaber AO, Moore LW, Alloway RR, et al. Acute rejection characteristics from a prospective, randomized, double-blind, placebocontrolled multicenter trial of early corticosteroid withdrawal. Transplantation. 2013;95:573-579.

23. Walsh RC, Brailey P, Girnita A, et al. Early and late acute antibodymediated rejection differ immunologically and in response to proteasome inhibition. Transplantation. 2011;91:1218-1226.

24. Evans RW, Applegate WH, Briscoe DM, et al. Cost-related immunosuppressive medication nonadherence among kidney transplant recipients. Clin J Am Soc Nephrol. 2010;5:2323-2328.

25. Rodrigo E, Segundo DS, Fernández-Fresnedo G, et al. Withinpatient variability in tacrolimus blood levels predicts kidney graft loss and donor-specific antibody development. Transplantation. 2016;100:2479-2485.

26. Taber DJ, Su Z, Fleming JN, et al. Tacrolimus trough concentration variability and disparities in African American kidney transplantation. Transplantation. 2017;101:2931-2938.

27. Butler JA, Roderick P, Mullee M, Mason JC, Peveler RC. Frequency and impact of nonadherence to immunosuppressants after renal transplantation: a systematic review. Transplantation. 2004;77:769-776.

28. Fine RN, Becker Y, De Geest S, et al. Nonadherence consensus conference summary report. Am J Transplant. 2009;9:35-41.

How to cite this article: Liu A, Woodside KJ, Augustine JJ, Sarabu N. Racial disparity in kidney transplant survival relates to late rejection and is independent of steroid withdrawal. Clin Transplant. 2018;32:e13381. https://doi.org/10.1111/ctr.13381 